\title{
Delayed surgery for localised and metastatic renal cell carcinoma: a systematic review and meta-analysis for the COVID-19 pandemic
}

\author{
Vinson Wai-Shun Chan ${ }^{1,3,8}$ (D) Wei Shen Tan ${ }^{2,3}$. Jeffrey J. Leow ${ }^{4,5} \cdot$ Wei Phin Tan ${ }^{6}$. William Lay Keat Ong ${ }^{7}$. \\ Peter Ka-Fung Chiu ${ }^{8}$. Pratik Gurung ${ }^{9}$. Giacomo Maria Pirola ${ }^{10}$. Luca Orecchia ${ }^{11}$. Matthew Ping Chao Liew ${ }^{12}$. \\ Hsiang-Ying Lee ${ }^{13} \cdot$ Yuding Wang ${ }^{14} \cdot$ I-Hsuan Alan Chen ${ }^{15}$. Daniele Castellani ${ }^{16} \cdot$ Marcelo Langer Wroclawski $^{17,18}$. \\ Nikhil Mayor ${ }^{19}$. Niranjan J. Sathianathen ${ }^{20}$. Isaac Braga ${ }^{21}$ - Zhenbang Liu ${ }^{4}$. Dora Moon ${ }^{22} \cdot$ Kari Tikkinen $^{23}$. \\ Ashish Kamat ${ }^{24} \cdot$ Max Meng $^{25}$ - Vincenzo Ficarra ${ }^{26}$. Gianluca Giannarini ${ }^{27}$. Jeremy Yuen-Chun Teoh ${ }^{8}$ (1)
}

Received: 17 March 2021 / Accepted: 13 May 2021 / Published online: 24 May 2021

(C) The Author(s), under exclusive licence to Springer-Verlag GmbH Germany, part of Springer Nature 2021

\begin{abstract}
Purpose The COVID-19 pandemic has led to the cancellation or deferment of many elective cancer surgeries. We performed a systematic review on the oncological effects of delayed surgery for patients with localised or metastatic renal cell carcinoma (RCC) in the targeted therapy (TT) era.

Method The protocol of this review is registered on PROSPERO(CRD42020190882). A comprehensive literature search was performed on Medline, Embase and Cochrane CENTRAL using MeSH terms and keywords for randomised controlled trials and observational studies on the topic. Risks of biases were assessed using the Cochrane RoB tool and the NewcastleOttawa Scale. For localised RCC, immediate surgery [including partial nephrectomy (PN) and radical nephrectomy (RN)] and delayed surgery [including active surveillance (AS) and delayed intervention (DI)] were compared. For metastatic RCC, upfront versus deferred cytoreductive nephrectomy $(\mathrm{CN})$ were compared.

Results Eleven studies were included for quantitative analysis. Delayed surgery was significantly associated with worse cancer-specific survival (HR 1.67, 95\% CI 1.23-2.27, $p<0.01$ ) in T1a RCC, but no significant difference was noted for overall survival. For localised $\geq$ T1b RCC, there were insufficient data for meta-analysis and the results from the individual reports were contradictory. For metastatic RCC, upfront TT followed by deferred CN was associated with better overall survival when compared to upfront CN followed by deferred TT (HR 0.61, 95\% CI $0.43-0.86, p<0.001$ ).

Conclusion Noting potential selection bias, there is insufficient evidence to support the notion that delayed surgery is safe in localised RCC. For metastatic RCC, upfront TT followed by deferred CN should be considered.
\end{abstract}

Keywords Renal cell carcinoma $\cdot$ Active surveillance $\cdot$ Delayed surgery $\cdot$ Targeted therapy $\cdot$ Nephrectomy $\cdot$ COVID-19

$\begin{array}{ll}\text { Abbreviations } \\ \text { RCC } & \text { Renal cell carcinoma } \\ \text { mRCC } & \text { Metastatic RCC } \\ \text { PN } & \text { Partial nephrectomy } \\ \text { AS } & \text { Active surveillance } \\ \text { DI } & \text { Delayed intervention } \\ \text { CN } & \text { Cytoreductive nephrectomy } \\ \text { OS } & \text { Overall survival } \\ \text { PFS } & \text { Progression-free survival } \\ \text { CSS } & \text { Cancer-specific survival } \\ \text { RFS } & \text { Recurrence-free survival }\end{array}$

Jeremy Yuen-Chun Teoh

jeremyteoh@surgery.cuhk.edu.hk

Extended author information available on the last page of the article

$\begin{array}{ll}\text { HR } & \text { Hazard ratio } \\ \text { 95\% CI } & 95 \% \text { Confidence interval } \\ \text { SEER } & \text { Surveillance, epidemiology and end results } \\ & \text { program } \\ \text { NCDB } & \text { National cancer database } \\ \text { SWT } & \text { Surgical waiting time }\end{array}$

\section{Introduction}

Historically, surgery has been the benchmark for the treatment of localised RCC. However, emerging evidence has shown comparable outcomes among various modalities such as partial nephrectomy (PN), tumour ablation and active surveillance (AS) for small renal masses [1, 
2]. In the metastatic RCC (mRCC) setting, data from CARMENA and SURTIME has questioned the role of cytoreductive nephrectomy with the exception of selected patients with low-risk oligometastatic disease [3, 4].

The delivery of urological services around the world has been severely disrupted during the COVID-19 pandemic $[5,6]$. While there has been considerable focus in the prevention and management of COVID-19 in both medical professionals and patients [7], the potential harm of delayed surgery for patients with RCC should not be under-estimated. Therefore, in this study, we systematically reviewed the literature and performed a meta-analysis to quantify the risk of delayed surgery in patients with localised RCC, and mRCC in the targeted therapy (TT) era. These results will provide valuable insight on how we should manage RCC during this difficult time period of COVID-19 and any further waves of infections.

\section{Methods and materials}

We performed a systematic review to investigate the effects of delayed surgery for patients with localised RCC and $\mathrm{mRCC}$ in the TT era. This review was performed according to the Preferred Reporting Items for Systematic Reviews and Meta-Analyses (PRISMA) statement [8] and the Meta-analyses Of Observational Studies in Epidemiology (MOOSE) guidelines [9]. This systematic review was written a priori on PROSPERO (International prospective register of systematic reviews) with the registration number CRD42020190882.

\section{Literature search}

A comprehensive literature search was performed using a combination of keywords (Medical Subject Headings $(\mathrm{MeSH})$ and free text words) including "Renal Cell Carcinoma", "Partial Nephrectomy", "Radical Nephrectomy", "Cytoreductive Nephrectomy", "Delay", "Postpone" and "active surveillance"; special features such as explosion were also utilised in the search to retrieve all records indexed to any narrower MeSH terms. The last search was performed on the 8th Jul 2020 on OVID MEDLINE, EMBASE and Cochrane Central Controlled Register of Trials (CENTRAL) by an experienced Cochrane author. No date limit was imposed on the search, while only articles written in English, or those containing an English abstract, were included. The search strategy is presented in Online Appendix 1. Additional articles were sought from the reference lists of the included articles.

\section{Selection criteria}

Patients with both localised and mRCC were included in this systematic review. For localised RCC, the immediate surgery group included patients who underwent $\mathrm{PN}$ or radical nephrectomy (RN), and the delayed surgery group included patients who underwent AS and delayed intervention (DI). AS was defined as the non-receipt of any surgical treatment undergoing active follow-up, while DI was defined as any deferred surgical treatment when compared to standard practice over the period of 3 months. Owing to clinical uncertainty and limited studies available to establish the standard use of ablation therapies [10], studies solely investigating these treatments were excluded from our analysis; however, there was no restriction to the type of surgical approach (i.e., open, laparoscopic or robotic) received by the RCC patients. For mRCC, only patients who received TT were included, and we compared patients who underwent upfront cytoreductive nephrectomy $(\mathrm{CN})$ followed by TT, with those who received upfront TT and deferred $\mathrm{CN}$. All observational comparative studies were included in this review. Conference abstracts, letters, editorials and single-arm studies were excluded. Finally, only studies reporting survival outcomes were included.

\section{Screening and data extraction}

All records identified in the literature search were initially screened by title and abstract by three independent and blinded reviewers (V.W.S.C, W.L.K.O, P.K.F.C), and conflicts were solved by senior author (J.Y.C.T). Full texts were then retrieved for further independent screening by two authors (V.W.S.C and W.L.K.O) against the selection criteria. The authors then independently performed data extraction and risk of bias assessment using a piloted, standardised form for data entry. Baseline characteristics, potential confounders, descriptions of intervention and control, and desired outcomes were collected.

\section{Data synthesis and statistical analysis}

The primary outcome of our study is the effects of delayed surgery on survival outcomes for RCC patients when compared to immediate surgical intervention. Survival outcomes included overall survival (OS), progression-free survival (PFS), cancer-specific survival (CSS) and recurrence-free survival (RFS) measured from the time of diagnosis or treatment. Survival was measured by hazard ratio (HR) and 95\% confidence interval (95\% CI) derived from Cox proportional hazards model. In studies where only the Kaplan-Meier survival plots were reported, the HR was extracted using 
Engauge Digitizer Software and Stata 16. Meta-analyses were only performed when there were three or more studies reporting the same outcome. Where multiple studies reported results from large prospectively maintained cancer databases such as the Surveillance, Epidemiology and End Results Program (SEER) and National Cancer Database (NCDB), the largest cohort for each outcome was used. Effects from individual eligible studies were pooled and analysed using Stata 16 (StataCorp LLC) and reported as HR, 95\% CI, weightings and forest plots; the random effects model and the restricted maximum-likelihood approach was used. Substantial heterogeneity was defined as an $I^{2}$ value $>50 \%$ or a Chi-square $p$ value $<0.10$. Where substantial heterogeneity was predicted or confirmed upon primary analyses, sensitivity analyses were performed. Sensitivity analyses were performed by subgroup analyses between different surgical approaches; and subgroup differences were defined as a Chi-square $p$ value $<0.10$. Sensitivity analyses were also performed on outcomes incorporating retrospective studies from SEER and NCDB as a result of potential heterogeneity in selection criteria and management of small renal masses. The Newcastle-Ottawa Scale was used to assess the risk of bias in non-randomised observational study; and the Cochrane Risk of bias 1.0 tool was used to assess the risk of bias in RCTs.

\section{Results}

The PRISMA flow diagram is shown in Supplementary Fig. 1A total of 3226 records were retrieved upon literature search. Six additional studies were identified through reference lists of the included articles. After 1186 duplicates were removed, 2046 records were screened by abstract and title. A total of 111 records were excluded upon full text screening, and the reasons are provided in Online Appendix 1. Twentyfour studies were included for qualitative synthesis. SEER studies with the largest patient cohort for each outcome were selected, hence four eligible studies were excluded for metaanalysis [11-14]. Finally, a total of 11 studies were included for meta-analysis. The risk of bias (RoB) assessments of these studies is shown in the Online Appendix 1.

\section{Immediate versus delayed surgery in localised RCC patients}

\section{Patients with T1a disease}

Six studies compared OS for AS, DI, PN and RN in T1a patients. The baseline characteristics and references of these studies are reported in Supplementary Table 1. Initial meta-analysis did not suggest a significantly different OS in patients undergoing AS or DI compared to those undergoing immediate PN and RN (HR 1.36, 95\% CI $0.99-1.87, p=0.06$; Fig. 1) with substantial heterogeneity $\left(I^{2}=87.07 \%, p<0.001\right)$. Hence heterogeneity was explored. Upon removal of large studies from the SEER database [15] and NCDB [16] which did not have standardised and consistent AS or DI protocols, significant heterogeneity no longer existed $\left(I^{2}=0.00 \%, p=0.38\right)$. AS and DI remained indifferent to PN and RN for OS (HR: 1.08, 95\% CI 0.83-1.40, $p=0.58$; Supplementary Fig. 1) with no subgroup differences $(p=0.71$ and 0.54$)$.

A total of two studies reported CSS after AS or DI. Incorporating 14,168 patients, delayed surgery was associated with worse CSS in T1a patients when compared to immediate nephrectomy (HR 1.67, 95\% CI 1.23-2.27, $p<0.01$; Supplementary Fig. 2) [15, 17]. Although substantial heterogeneity within studies investigating AS was noted, more robust subgroup analysis was not possible due to limited number of studies. For DI, in a cohort of 292 patients, 32 patients underwent delayed surgery with a mean observation period of 26.2 months (range: 6.5-74.8 months). DI was associated with significantly worsened CSS $(p<0.001)$ but not worsened OS and RFS [18].

\section{Patients with $\mathrm{T} 1 \mathrm{~b}$ or above disease}

Three studies reported survival outcomes in stage $\geq \mathrm{T} 1 \mathrm{~b}$ or above disease. Mano et al. showed that longer surgical waiting time (SWT) as a continuous variable was significantly associated with worse OS, but not CSS (HR 1.17, 95\% CI 1.08-1.27, $p<0.001$; HR 0.94, 95\% CI 0.80-1.11, $p=0.5$, respectively) [19]. Shiff et al. categorised waiting times into 4-8 weeks, 8-12 weeks and 12-24 weeks, and found that OS, RFS and CSS were not significantly worsened when compared to those received treatment within 4 weeks upon multivariable analysis [20]. For patients who had surgery delayed for over 12-24 weeks were, however, at risk of worsened RFS (HR 0.50, 95\% CI 0.30-0.83, $p=0.01$ ) only upon univariate analysis [20]. A recent NCDB study by Srivastava et al. have, however, found 1-3 months and 3 months or more wait to surgery to worsen OS in T1b patients when compared to patients undergoing immediate surgery during multivariate analysis. (HR 1.13, 95\% CI 1.04-1.22, $p<0.001$; HR $1.55,95 \%$ CI 1.49-1.73, $p<0.001$, respectively) [21]. The outcomes of $\mathrm{T} 1$ disease in general are reported qualitatively in Online Appendix 1.

\section{Patients with $\mathrm{T} 2$ or above disease}

Three studies investigating the effect of delayed surgery in patients with $\mathrm{T} 2$ or above disease were found. Kim et al. showed that SWT as a continuous variable did not affect RFS and CSS in patients undergoing RN [22]. The results did not differ when stratified to T2 or T3-4 patients alone [22]. 


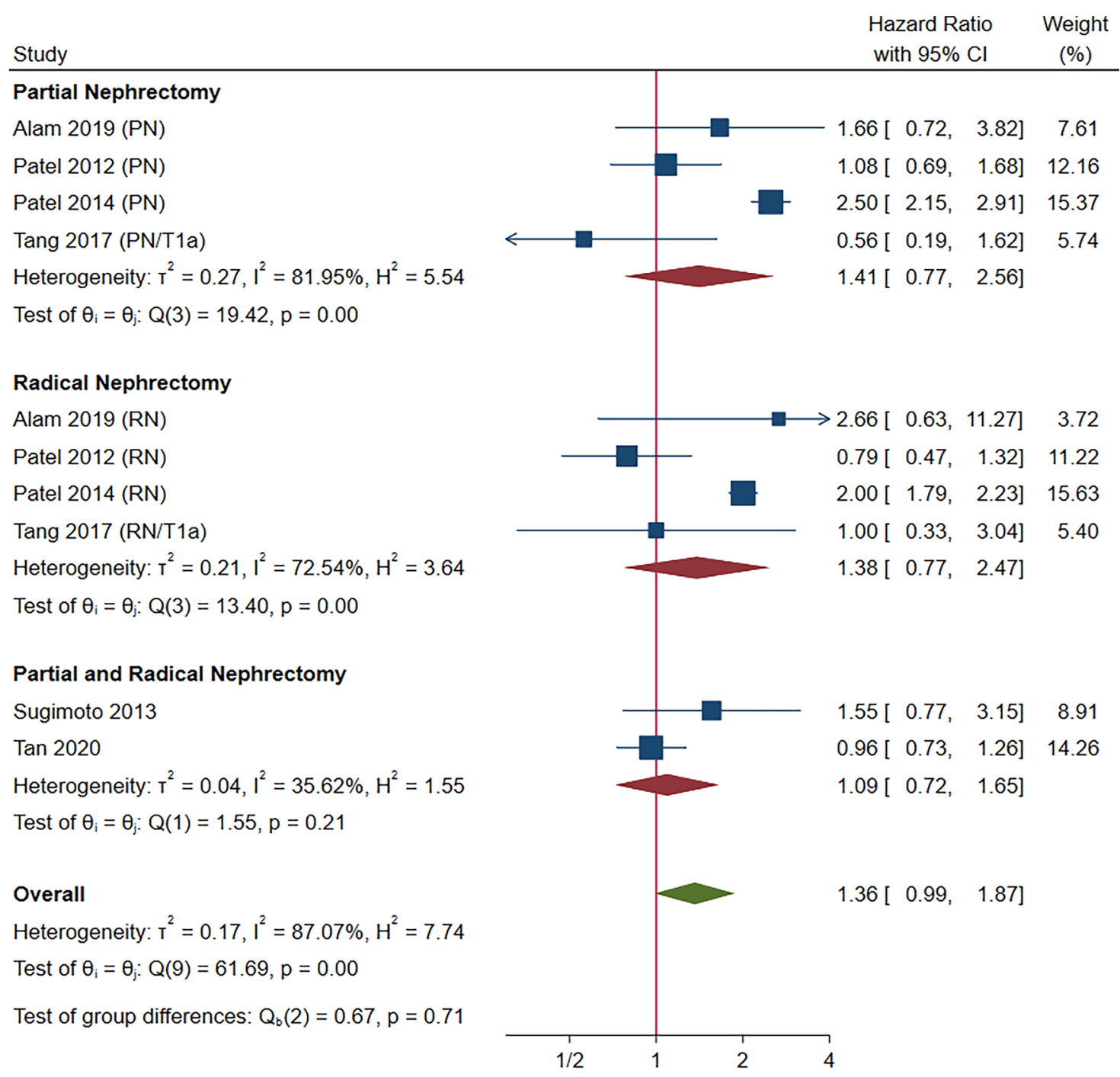

Random-effects REML model

Fig. 1 Overall survival in T1a renal cell carcinoma (pre-sensitivity analysis)

Similarly, on multivariable analysis Srivastava et al. did not find a 1-3-month or over 3-month delay to worsen OS when compared to immediate intervention in cT2a and cT2b patients [21]. In a large Canadian registry study published by Shiff et al., also did not find any delay up to 24 weeks to worsen RFS, CSS and OS in cT2 tumours; equally, RFS and OS is not worsened in cT3/4 patients in a delay up to 24 weeks [20]. Outcomes for patients with any stages of localised RCC are summarized qualitatively in the supplementary appendix. No studies investigating delay surgery in locally advanced RCC patients were identified.

\section{Sequencing of cytoreductive nephrectomy and targeted therapy in $\mathrm{mRCC}$ patients}

Three retrospective studies and one RCT investigated the sequence of $\mathrm{CN}$ and TT for mRCC. The baseline characteristics and references of these studies are reported in Supplementary Table 2. Our meta-analysis concluded a significantly improved OS amongst patients receiving upfront TT followed by $\mathrm{CN}$ when compared to those received upfront CN followed by TT (HR 0.61, 95\% CI 0.44-0.86, $p<0.001$; Fig. 2). While substantial heterogeneity $\left(I^{2} 73.0 \%, p<0.01\right)$ 


\begin{tabular}{l} 
Study \\
\hline Bex 2019 \\
Bhindi 2020 \\
Hanna 2016 \\
Stroup 2013 \\
Overall \\
Heterogeneity: $\mathrm{T}^{2}=0.06, \mathrm{I}^{2}=67.72 \%, \mathrm{H}^{2}=3.10$ \\
Test of $\theta_{\mathrm{i}}=\theta_{\mathrm{j}}: \mathrm{Q}(3)=12.39, \mathrm{p}=0.01$ \\
Test of $\theta=0: \mathrm{z}=-3.01, \mathrm{p}=0.00$ \\
\end{tabular}

Random-effects REML model

Fig. 2 Overall survival in metastatic renal cell carcinoma (pre-sensitivity analysis)

were found, the direction of effect is the same in all study, with potential heterogeneity originated from the effects of large sample size reported by Hanna et al.

PFS was only reported in one RCT-the SURTIME trial [4]. There was no significant difference in PFS amongst those who received $\mathrm{CN}$ before TT and those received TT before CN (HR 0.87, 95\% CI 0.56-1.37, $p=0.57$ ) [4].

\section{Discussion}

Data regarding the effects of delayed surgical intervention in kidney cancer are still limited. Our present study reviewed the survival outcomes of active surveillance (AS), delayed intervention (DI) and surgical therapy in patients with localised RCC and $\mathrm{mRCC}$ in the TT era.

For patients with T1a localised RCC, most studies showed comparable survival outcomes of patients managed with AS and DI compared to those treated with immediate surgeries [23-25]. This is particularly reflected in the carefully selected patients with advanced age in AS cohorts. Surgical management with $\mathrm{PN}$ or RN might be preferred in younger patients, regardless of the disease stages. As the difference in all-course mortality between the treatment group versus nonsurgical group might be attributed to selection bias, we must take both patient and disease characteristics into account and make a balance between the potential benefit and harm of undergoing surgery. The European Association of Urology (EAU) COVID-19 guidelines recommend treatment to be deferred for 6 months after diagnosis for asymptomatic cT1a patients [26].

To date, there are few studies assessing the effect of delayed surgery for $\geq \mathrm{T} 1 \mathrm{~b}$ RCC and data is less robust. While we found CSS and RFS not to be worsened in patients with delayed surgery for $\geq \mathrm{T} 1 \mathrm{~b} \mathrm{RCC}$, results are contradicting in terms of OS [19, 27]. Worsen OS could be a result of potential delay due to confounding bias and patient optimisation due to multiple comorbidities, hence deaths were unlikely to be oncological related. In summary, data on the effects of delayed surgery in T1b or above kidney tumours are limited, contradictory and prone to selection bias. Delay in surgery is potentially harmful in terms of cancer control and early surgery should be offered despite the risk of COVID-19. The EAU COVID-19 guidelines recommend treatment within 3 months for asymptomatic cT1b to cT2a patients, while more advanced RCCs should be treated within six weeks [26], however, the evidence for delayed surgery in locally advanced disease is very limited.

In regard to $\mathrm{mRCC}$, the timing of $\mathrm{CN}$ and its traditional role has been challenged with the advent of latest tyrosine kinase inhibitors and immune checkpoint inhibitors [28]. The pivotal SURTIME trial provides evidence for delayed CN over immediate surgery [4]. Our meta-analysis also concluded that there was a significant improvement of OS in patients receiving upfront TT followed by $\mathrm{CN}$ compared to those undergoing upfront $\mathrm{CN}$ followed by TT (HR 0.61, 95\% CI $0.44-0.84, p<0.01)$. Hence, it is in line with this landmark study that systemic treatment should be given upfront for mRCC before consideration of CN [4]. Patient selection for $\mathrm{CN}$ based on established risk stratification is of paramount importance and multimodal treatment remains critical for the management of mRCC [29].

In the current climate of COVID-19, prioritisation of elective surgery, including urological cancer treatment is inevitable and necessary [30-32]. The assessment of risk in delaying any procedures must be made considering patient safety as well as potential adverse effects on oncological outcomes. In this review, we concluded that there is insufficient data to support the notion that delayed surgery is safe in patients with localised RCC. For mRCC patients, it is 
also desirable to initiate TT upfront followed by deferred CN. Recognising the lack of data in this area, the COVID19 pandemic serves as a good opportunity to understand the effects of SWT in RCC patients who would typically undergo early surgery [33].

Our study includes review of one RCT, one prospective non-randomised trial and six retrospective cohorts. This study is limited by retrospective cohorts, with small population numbers and potential inherent biases. These small population does not allow for subgroup analyses of different surgical approaches such as open, laparoscopic or robotic cases, which may introduce a certain degree of bias. Furthermore, case selection and confounding bias may be unadjusted in the included studies suggesting unequal risks of non-oncological related death. Finally, owing to clinical uncertainties studies solely assessing ablative therapies were not included. However, we recognised that they could be alternatives to surgical management during the COVID19 pandemic as ablative therapies could potentially avoid risks associated with general anaesthesia to both the patient and healthcare workers, if routinely performed under local anaesthetics. Despite these limitations, this study provides a valuable synthesis of the current literature and interim guidance regarding the management of RCC in the pandemic of COVID-19. The results also highlight the need for highquality research in the future.

\section{Conclusion}

Delayed surgery was associated with worse CSS but not OS in T1a RCC, which could be a result of significant selection bias in included studies. Results on T1b or above disease and locally advanced disease were limited and contradictory. Despite the risk of COVID-19 infection, early surgery should be considered to optimise the oncological outcomes in localised RCC. For mRCC, initial TT followed by deferred $\mathrm{CN}$ was associated with improved OS. Our results provide interim guidance regarding the management of RCC during the pandemic of COVID-19. More high-quality studies will be needed in this area.

Supplementary Information The online version contains supplementary material available at https://doi.org/10.1007/s00345-021-03734-1.

Author contributions Vinson Wai-Shun Chan contributed to proto$\mathrm{col} /$ project development, data collection or management, data analysis and manuscript writing/editing. Wei Shen Tan contributed to protocol/ project development, data collection, and manuscript writing/editing. Jeffrey Leow contributed to protocol/project development, data collection, and manuscript writing/editing. Wei Phin Tan contributed to protocol/project development, data collection, and manuscript writing/ editing. William Lay Keat Ong contributed to protocol/project development, data collection, and manuscript writing/editing. Peter Ka-Fung Chiu contributed to protocol/project development, data collection, and manuscript writing/editing. Pratik Gurung contributed to protocol/ project development, data collection, and manuscript writing/editing. Giacomo Maria Pirola contributed to protocol/project development, data collection, and manuscript writing/editing. Luca Orecchia contributed to protocol/project development, data collection, and manuscript writing/editing. Matthew Ping Chao Liew contributed to protocol/ project development, data collection, and manuscript writing/editing. Hsiang-Ying Lee contributed to protocol/project development, data collection, and manuscript writing/editing. Yuding Wang contributed to protocol/project development, data collection, and manuscript writing/ editing. I-Hsuan Alan Chen contributed to protocol/project development, data collection, and manuscript writing/editing. Daniele Castellani contributed to protocol/project development, data collection, and manuscript writing/editing. Marcelo Langer Wroclawski contributed to protocol/project development, data collection, and manuscript writing/editing. Nikhil Mayor contributed to protocol/project development, data collection, and manuscript writing/editing. Niranjan J. Sathianathen contributed to protocol/project development, data collection, and manuscript writing/editing. Isaac Braga contributed to protocol/project development, data collection, and manuscript writing/editing. Zhenbang Liu contributed to protocol/project development, data collection, and manuscript writing/editing. Dora Moon contributed to protocol/ project development, data collection, and manuscript writing/editing. Kari Tikkinen contributed to protocol/project development, data collection, and manuscript writing/editing. Ashish Kamat contributed to protocol/project development, data collection, and manuscript writing/ editing. Max Meng contributed to protocol/project development, data collection, and manuscript writing/editing. Vincenzo Ficarra contributed to protocol/project development, data collection, and manuscript writing/editing. Gianluca Giannarini contributed to protocol/project development, data collection, and manuscript writing/editing. Jeremy Yuen-Chun Teoh contributed to protocol/project development, data collection or management, data analysis and manuscript writing/editing.

Funding No funding was received for conducting this study. The authors have no relevant financial or non-financial interests to disclose.

\section{Declaration}

Ethical approval As this is a secondary study of already published studies, no informed consent or ethical approvals are required.

\section{References}

1. Andrews JR, Atwell T, Schmit G, Lohse CM, Kurup AN, Weisbrod A, Callstrom MR, Cheville JC, Boorjian SA, Leibovich BC, Thompson RH (2019) Oncologic outcomes following Partial Nephrectomy and percutaneous Ablation for cT1 renal masses. Eur Urol 76(2):244-251. https://doi.org/10.1016/j.eururo.2019. 04.026

2. Simone G, De Nunzio C, Ferriero M, Cindolo L, Brookman-May S, Papalia R, Sperduti I, Collura D, Leonardo C, Anceschi U, Tuderti G, Misuraca L, Dalpiaz O, Hatzl S, Lodde M, Trenti E, Pastore AL, Palleschi G, Lotrecchiano G, Salzano L, Carbone A, De Cobelli O, Tubaro A, Schips L, Zigeuner R, Tostain J, May M, Guaglianone S, Muto G, Gallucci M (2016) Trends in the use of partial nephrectomy for cT1 renal tumors: analysis of a 10-yr European multicenter dataset. Eur J Surg Oncol 42(11):17291735. https://doi.org/10.1016/j.ejso.2016.03.022

3. Méjean A, Ravaud A, Thezenas S, Colas S, Beauval J-B, Bensalah K, Geoffrois L, Thiery-Vuillemin A, Cormier L, Lang H, Guy L, Gravis G, Rolland F, Linassier C, Lechevallier E, Beisland C, Aitchison M, Oudard S, Patard J-J, Theodore C, 
Chevreau C, Laguerre B, Hubert J, Gross-Goupil M, Bernhard J-C, Albiges L, Timsit M-O, Lebret T, Escudier B (2018) Sunitinib alone or after Nephrectomy in metastatic renal-cell carcinoma. N Engl J Med 379(5):417-427. https://doi.org/10.1056/ NEJMoa1803675

4. Bex A, Mulders P, Jewett M, Wagstaff J, Van Thienen JV, Blank CU, Van Velthoven R, Del Pilar LM, Wood L, Van Melick HHE, Aarts MJ, Lattouf JB, Powles T, De Jong IJ, Rottey S, Tombal B, Marreaud S, Collette S, Collette L, Haanen J (2019) Comparison of immediate vs deferred cytoreductive Nephrectomy in patients with synchronous metastatic renal cell carcinoma receiving Sunitinib: the SURTIME Randomized Clinical Trial. JAMA Oncol 5(2):164-170. https://doi.org/10.1001/jamaoncol.2018.5543

5. Teoh JY-C, Ong WLK, Gonzalez-Padilla D, Castellani D, Dubin JM, Esperto F, Campi R, Gudaru K, Talwar R, Okhunov Z, Ng C-F, Jain N, Gauhar V, Wong MC-S, Wroclawski ML, Tanidir Y, Rivas JG, Tiong H-Y, Loeb S, UroSoMe Working G (2020) A global survey on the impact of COVID-19 on urological services. Eur Urol 78(2):265-275. https://doi.org/10.1016/j.eururo.2020.05. 025

6. Ong WLK, Lechmiannandan S, Loeb S, Teoh JY (2020) Urological services in public hospitals suffered a greater detriment than private hospitals during the battle of COVID-19. Urology. https:// doi.org/10.1016/j.urology.2020.07.010

7. Esperto F, Prata F, Civitella A, Pang KH, Marchioni M, Tuzzolo P, Scarpa RM, Papalia R (2020) Implementation and strategies to ensure adequate coordination within a Urology Department during the COVID-19 pandemic. International Braz J Urol : official journal of the Brazilian Society of Urology 46 (suppl.1):170-180. https://doi.org/10.1590/S1677-5538.IBJU.2020.S122

8. Moher D, Liberati A, Tetzlaff J, Altman DG (2009) Preferred reporting items for systematic reviews and meta-analyses: the PRISMA statement. BMJ 339:b2535. https://doi.org/10.1136/ bmj.b2535

9. Stroup DF, Berlin JA, Morton SC, Olkin I, Williamson GD, Rennie D, Moher D, Becker BJ, Sipe TA, Thacker SB (2000) Metaanalysis of observational studies in epidemiology: a proposal for reporting. Meta-analysis Of Observational Studies in Epidemiology (MOOSE) group. JAMA 283 (15):2008-2012. https://doi.org/ 10.1001/jama.283.15.2008

10. Abu-Ghanem Y, Fernández-Pello S, Bex A, Ljungberg B, Albiges L, Dabestani S, Giles RH, Hofmann F, Hora M, Kuczyk MA, Kuusk T, Marconi L, Merseburger AS, Tahbaz R, Staehler M, Volpe A, Powles T, Lam TB, Bensalah K (2020) Limitations of available studies prevent reliable comparison between tumour Ablation and Partial Nephrectomy for patients with localised renal masses: a systematic review from the European Association of Urology Renal Cell Cancer Guideline Panel. Eur Urol Oncol 3(4):433-452. https://doi.org/10.1016/j.euo.2020.02.001

11. Xing M, Kokabi N, Zhang D, Ludwig JM, Kim HS (2018) Comparative effectiveness of thermal ablation, surgical resection, and active surveillance for t1a renal cell carcinoma: a Surveillance, Epidemiology, and End Results (SEER)-Medicare-linked Population Study. Radiology 288(1):81-90. https://doi.org/10.1148/ radiol.2018171407

12. Patel HD, Kates M, Pierorazio PM, Allaf ME (2015) Balancing cardiovascular (CV) and cancer death among patients with small renal masses: modification by CV risk. BJU Int 115(1):58-64. https://doi.org/10.1111/bju.12719

13. Patel HD, Kates M, Pierorazio PM, Gorin MA, Jayram G, Ball MW, Hyams ES, Allaf ME (2014) Comorbidities and causes of death in the management of localized T1a kidney cancer. Int $\mathbf{J}$ Urol 21(11):1086-1092. https://doi.org/10.1111/iju.12527

14. Sun M, Becker A, Tian Z, Roghmann F, Abdollah F, Larouche A, Karakiewicz PI, Trinh QD (2014) Management of localized kidney cancer: calculating cancer-specific mortality and competing risks of death for surgery and nonsurgical management. Eur Urol 65(1):235-241. https://doi.org/10.1016/j.eururo.2013.03.034

15. Patel HD, Kates M, Pierorazio PM, Hyams ES, Gorin MA, Ball MW, Bhayani SB, Hui X, Thompson CB, Allaf ME (2014) Survival after diagnosis of localized T1a kidney cancer: current population-based practice of surgery and nonsurgical management. Urology 83(1):126-132. https://doi.org/10.1016/j.urology.2013. 08.088

16. Tan WS, Trinh QD, Hayn MH, Marchese M, Lipsitz SR, Nabi J, Kilbridge KL, Vale JA, Khoubehi B, Kibel AS, Sun M, Chang SL, Sammon JD (2020) Delayed nephrectomy has comparable longterm overall survival to immediate nephrectomy for cT1a renal cell carcinoma: a population-based analysis. Urol Oncol 38(3):74. https://doi.org/10.1016/j.urolonc.2019.11.008

17. Becker A, Roghmann F, Ravi P, Tian Z, Kluth LA, Gandaglia G, Noldus J, Dahlem R, Schlomm T, Graefen M, Karakiewicz PI, Trinh QD, Sun M (2014) Delay in nephrectomy and cancer control outcomes in elderly patients with small renal masses. Urol Int 92(4):455-461. https://doi.org/10.1159/000356175

18. Sugimoto K, Shimizu N, Nose K, Tahara H, Imanishi M, Nishioka T, Esa A, Kajikawa H, Uemura H (2013) Clinical outcome of small renal cell carcinoma after delayed surgery versus immediate surgery. J Cancer 4(6):514-518. https://doi.org/10.7150/jca.6949

19. Mano R, Vertosick EA, Hakimi AA, Sternberg IA, Sjoberg DD, Bernstein M, Dalbagni G, Coleman JA, Russo P (2016) The effect of delaying nephrectomy on oncologic outcomes in patients with renal tumors greater than $4 \mathrm{~cm}$. Urol Oncol 34(5):239. https://doi. org/10.1016/j.urolonc.2015.12.001

20. Shiff B, Breau RH, Patel P, Mallick R, Tanguay S, So A, Lavallée L, Moore R, Rendon R, Kapoor A, Pouliot F, Finelli A, Bhindi B, Lattouf J-B, Basappa N, Wood L, Heng D, Bjarnason $\mathrm{G}$, Drachenberg D Impact of time-to-surgery and surgical delay on oncologic outcomes for renal cell carcinoma. Journal of Urology 0 (0):https://doi.org/10.1097/JU.0000000000001230.

21. Srivastava A, Patel HV, Kim S, Shinder B, Sterling J, Tabakin AL, Polotti CF, Saraiya B, Mayer T, Kim IY, Ghodoussipour S, Patel HD, Jang TL, Singer EA (2020) Delaying surgery for clinical T1b-T2bN0M0 renal cell carcinoma: Oncologic implications in the COVID-19 era and beyond. Urol Oncol. https://doi.org/10. 1016/j.urolonc.2020.10.012

22. Kim KH, You D, Jeong IG, Song C, Hong JH, Ahn H, Kim CS (2012) The impact of delaying radical nephrectomy for stage II or higher renal cell carcinoma. J Cancer Res Clin Oncol 138(9):1561-1567. https://doi.org/10.1007/s00432-012-1230-2

23. Alam R, Patel HD, Osumah T, Srivastava A, Gorin MA, Johnson MH, Trock BJ, Chang P, Wagner AA, McKiernan JM, Allaf ME, Pierorazio PM (2019) Comparative effectiveness of management options for patients with small renal masses: a prospective cohort study. BJU Int 123(1):42-50. https://doi.org/10.1111/bju.14490

24. Patel N CD, Akhtar M. Z, George C, Jones A, Leiblich A, Protheroe A, Sullivan M. (2012) Active surveillance of small renal masses offers short-term oncological efficacy equivalent to radical and partial nephrectomy.

25. Tang DH, Nawlo J, Chipollini J, Gilbert SM, Poch M, Pow-Sang JM, Sexton WJ, Spiess PE (2017) Management of renal masses in an Octogenarian cohort: is there a right approach? Clin Genitourin Cancer 15(6):696-703. https://doi.org/10.1016/j.clgc.2017.05.011

26. Ribal MJ, Cornford P, Briganti A, Knoll T, Gravas S, Babjuk M, Harding C, Breda A, Bex A, Group G, Rassweiler JJ, Gözen AS, Pini G, Liatsikos E, Giannarini G, Mottrie A, Subramaniam R, Sofikitis N, Rocco BMC, Xie L-P, Witjes JA, Mottet N, Ljungberg B, Rouprêt M, Laguna MP, Salonia A, Bonkat G, Blok BFM, Türk C, Radmayr C, Kitrey ND, Engeler DS, Lumen N, Hakenberg OW, Watkin N, Hamid R, Olsburgh J, Darraugh J, Shepherd R, Smith E-J, Chapple CR, Stenzl A, Van Poppel H, Wirth M, Sønksen J, N’Dow J, Offices EAUS, the EAUGP (2020) 
European Association of Urology Guidelines Office Rapid Reaction Group: An Organisation-wide Collaborative Effort to Adapt the European Association of Urology Guidelines Recommendations to the Coronavirus Disease 2019 Era. European urology 78 (1):21-28. https://doi.org/10.1016/j.eururo.2020.04.056

27. Mano R, Vertosick EA, Hakimi AA, Sternberg IA, Sjoberg DD, Bernstein M, Dalbagni G, Coleman JA, Russo P (2016) The effect of delaying nephrectomy on oncologic outcomes in patients with renal tumors greater than $4 \mathrm{~cm}$. Urol Oncol 34 (5):239 e231-238. https://doi.org/10.1016/j.urolonc.2015.12.001

28. Ljungberg B, Albiges L, Abu-Ghanem Y, Bensalah K, Dabestani S, Fernandez-Pello S, Giles RH, Hofmann F, Hora M, Kuczyk MA, Kuusk T, Lam TB, Marconi L, Merseburger AS, Powles T, Staehler M, Tahbaz R, Volpe A, Bex A (2019) European Association of Urology Guidelines on renal cell carcinoma: the 2019 update. Eur Urol 75(5):799-810. https://doi.org/10.1016/j.eururo. 2019.02.011

29. Kapoor A, Wong ECL, Fang W, Wong NC (2019) Upfront cytoreductive nephrectomy vs. upfront systemic therapy in metastatic kidney cancer. Can Urol Assoc J:E377-E381. https://doi.org/10. 5489/cuaj.5666

30. Heldwein FL, Loeb S, Wroclawski ML, Sridhar AN, Carneiro A, Lima FS, Teoh JY (2020) A systematic review on guidelines and recommendations for urology standard of care during the COVID19 pandemic. Eur Urol Focus. https://doi.org/10.1016/j.euf.2020. 05.020

31. Ribal MJ, Cornford P, Briganti A, Knoll T, Gravas S, Babjuk M, Harding C, Breda A, Bex A, Group G, Rassweiler JJ, Gozen AS,
Pini G, Liatsikos E, Giannarini G, Mottrie A, Subramaniam R, Sofikitis N, Rocco BMC, Xie LP, Witjes JA, Mottet N, Ljungberg B, Roupret M, Laguna MP, Salonia A, Bonkat G, Blok BFM, Turk C, Radmayr C, Kitrey ND, Engeler DS, Lumen N, Hakenberg OW, Watkin N, Hamid R, Olsburgh J, Darraugh J, Shepherd R, Smith EJ, Chapple CR, Stenzl A, Van Poppel H, Wirth M, Sonksen J, N'Dow J, Offices EAUS, the EAUGP (2020) European Association of Urology Guidelines Office Rapid Reaction Group: An Organisation-wide Collaborative Effort to Adapt the European Association of Urology Guidelines Recommendations to the Coronavirus Disease 2019 Era. Eur Urol 78 (1):21-28. https://doi. org/10.1016/j.eururo.2020.04.056

32. Chan VW-S, Chiu PK-F, Yee C-H, Yuan Y, Ng C-F, Teoh JY-C (2020) A systematic review on COVID-19: urological manifestations, viral RNA detection and special considerations in urological conditions. World J Urol:1-12.https://doi.org/10.1007/ s00345-020-03246-4

33. Collaborative $\mathrm{C}$ Elective surgery cancellations due to the COVID19 pandemic: global predictive modelling to inform surgical recovery plans. BJS (British Journal of Surgery) n/a (n/a). https:// doi.org/10.1002/bjs.11746

Publisher's Note Springer Nature remains neutral with regard to jurisdictional claims in published maps and institutional affiliations.

\section{Authors and Affiliations}

\section{Vinson Wai-Shun Chan ${ }^{1,3,8}$ - Wei Shen Tan ${ }^{2,3}$. Jeffrey J. Leow ${ }^{4,5}$. Wei Phin Tan ${ }^{6}$. William Lay Keat Ong ${ }^{7}$. Peter Ka-Fung Chiu ${ }^{8}$. Pratik Gurung ${ }^{9}$. Giacomo Maria Pirola ${ }^{10}$. Luca Orecchia ${ }^{11}$ - Matthew Ping Chao Liew ${ }^{12}$. Hsiang-Ying Lee ${ }^{13} \cdot$ Yuding Wang ${ }^{14} \cdot$ I-Hsuan Alan Chen ${ }^{15}$. Daniele Castellani ${ }^{16}$. Marcelo Langer Wroclawski ${ }^{17,18}$. Nikhil Mayor ${ }^{19}$. Niranjan J. Sathianathen ${ }^{20}$. Isaac Braga ${ }^{21} \cdot$ Zhenbang Liu $^{4}$. Dora Moon ${ }^{22} \cdot$ Kari Tikkinen $^{23}$. Ashish Kamat ${ }^{24}$. Max Meng ${ }^{25}$. Vincenzo Ficarra ${ }^{26}$. Gianluca Giannarini ${ }^{27}$. Jeremy Yuen-Chun Teoh ${ }^{8} \mathbb{D}$}

1 School of Medicine, Faculty of Medicine and Health, University of Leeds, Leeds, UK

2 Department of Urology, Royal Free Hospital, London, UK

3 Division of Surgery and Interventional Science, University College London, London, UK

4 Department of Urology, Tan Tock Seng Hospital, Singapore, Singapore

5 Department of Urology, Lee Kong Chian School of Medicine, Nanyang Technological University, Singapore, Singapore

6 Department of Urology, Rush University Medical Center, Chicago, IL, USA

7 Department of Urology, Penang General Hospital, Penang, Malaysia

8 S.H. Ho Urology Centre, Department of Surgery, Prince of Wales Hospital, The Chinese University of Hong Kong, Hong Kong, China

9 Department of Urology, University of Rochester Medical Center, New York, USA

10 Urology Unit, San Donato Hospital, Arezzo, Italy
11 Urology Unit, Policlinico Tor Vergata Foundation, Rome, Italy

12 Department of Urology, Wrightington, Wigan and Leigh NHS Foundation Trust, Wigan, UK

13 Urology Department, Kaohsiung Municipal Ta-Tung Hospital, Kaohsiung, Taiwan

14 Division of Urology, Department of Surgery, McMaster University, Hamilton, Canada

15 Division of Urology, Department of Surgery, Kaohsiung Veterans General Hospital, Kaohsiung, Taiwan

16 Urology Division, Azienda Ospedaliero-Universitaria Ospedali Riuniti di Ancona, Università Politecnica delle Marche, Ancona, Italy

17 Hospital Israelita Albert Einstein, Sao Paulo, Brazil

18 BP-A Beneficencia Portuguesa de Sao Paulo, Sao Paulo, Brazil

19 Department of Urology, Royal Surrey County Hospital, Guildford, UK

20 Department of Urology, University of Melbourne, Parkville, Victoria, Australia 
21 Department of Urology, Instituto Portugues de Oncologia, Porto, Portugal

22 Department of Urology, East Lancashire Hospitals NHS Foundation Trust, Blackburn, UK

23 Departments of Urology and Public Health, University of Helsinki and Helsinki University Hospital, Helsinki, Finland

24 Department of Genitourinary Medical Oncology, The University of Texas MD Anderson Cancer Center, Houston, TX, USA
25

Department of Urology, University of California San Francisco, San Francisco, USA

26 Department of Human and Pediatric Pathology "Gaetano Barresi”, Urologic Section, University of Messina, Messina, Italy

27 Urology Unit, Santa Maria della Misericordia Academic Medical Centre, Udine, Italy 